\title{
Wahlprogramme im Vergleich
}

\author{
Sandra Brunsbach, Stefanie John, Andrea Volkens, Annika Werner
}

\section{Einleitung}

Die im Zuge der ersten Europawahlen entwickelte Nebenwahlthese von (K. Reif/H. Schmitt 1980: 130) postuliert für die Nachfrageseite der Politik eine geringere Relevanz der Europawahlen im Vergleich zu Wahlen der nationalen Parlamente. Hieraus ergibt sich eine Reihe von Konsequenzen für das Wahlverhalten der Bevölkerung und damit für die Wahlergebnisse: Erstens folge aus dem geringeren Interesse der Bevölkerung an Europawahlen eine niedrige Wahlbeteiligung. Zweitens seien Erfolge kleiner und neuer Parteien zu erwarten, da Wähler bei Nebenwahlen experimentierfreudiger seien bzw. eher ihren wahren Parteipräferenzen folgten, da keine Regierungsbildung zu verantworten sei. Drittens seien Wähler bei diesen Wahlen eher bereit, ihrer Unzufriedenheit mit den Parteien und mit der Politikumsetzung der Regierungsparteien Ausdruck zu verleihen. Daraus könnten sich zum einen ein höherer Anteil ungültiger Stimmen und zum anderen Wahlverluste insbesondere für Regierungsparteien ergeben (K. Reif/H. Schmitt 1980: 9-10). Diese Argumentation beruht auf dem Grundgedanken, dass die dominante Ebene - also die nationalstaatliche mit ihren Inhalten und Rationalitäten die Entscheidungsfindung der Wähler bei den Nebenwahlen determiniert.

Die Nebenwahlthese wurde hinsichtlich der zugrunde liegenden Thesen zu den Motivationen der Wähler durchaus kontrovers diskutiert. Obschon einige Studien alternative Erklärungsmodelle für das Wahlverhalten in Europawahlen aufzeigen (F. Ferrara/J. T. Weishaupt 2004) und die Erklärungen für das gute Abschneiden kleiner und neuer Parteien sowie für die Verluste von Regierungsparteien umstritten sind (C. Van der Eijk/M. N. Franklin 1996; A. Heath et al. 1999; T. Weber 2007), können die von Reif und Schmitt postulierten Charakteristika von Europawahlen für die westeuropäischen Mitgliedsstaaten weitgehend als bestätigt gelten. Für die Nachfrageseite wurde der Nebenwahlcharakter in einer Vielzahl von Studien dargelegt (u. a. H. Schmitt 1996; M. Marsh 1998, 2005; S. Hix/M. Marsh 2007). Trotz der Kompetenzerweiterung des Europäischen Parlamentes und der sukzessiven Ausweitung europäischer 
Rechtsetzung zeigen Europawahlen zumindest in Westeuropa weiterhin den Charakter von Nebenwahlen (H. Schmitt 2005; W. van der Brug et al. 2007) und entfalten in der Wahrnehmung der Wähler auch im Vergleich zu Regionaloder Kommunalwahlen eine geringere Relevanz (A. Heath et al. 1999; D. Schneider/P. Rössler 2005).

Eine systematische Betrachtung des Nebenwahlcharakters von Europawahlen auf der Angebotsseite liegt hingegen nur in Ansätzen vor. Zahlreiche Studien untersuchen die programmatischen Angebote von Parteien bei Europawahlen mit unterschiedlicher Schwerpunktsetzung, beziehen sich dabei allerdings höchstens indirekt auf den Nebenwahlcharakter. So werden beispielsweise verschiedene Modelle des europäischen Parteienwettbewerbs getestet (M. J. Gabel/S. Hix 2002) oder die Positionsveränderungen von Parteien zur EU im Zeitverlauf betrachtet (S. Kritzinger et al. 2004). Studien im Kontext des „Euromanifesto Projects“" (D. Braun et al. 2007) versuchen mit einer erweiterten Version des Kodierschemas der Manifesto Research Group (I. Budge et al. 2001) das sogenannte Framing von Parteien zu erfassen, indem sie die angesprochenen Kompetenzebenen berücksichtigen (A. M. Wüst/A. Volkens 2003: 6). Diese Untersuchungen stellen die (normativen) Zuordnungen der Parteien heraus, können diese jedoch nicht von der tatsächlichen Kompetenzzuordnung abgrenzen (A. M. Wüst/H. Schmitt 2007: 92). Für die deutschen Parteien belegen die Ergebnisse zwischen 1979 und 1999 eine Zunahme des europäischen Framings der Themen in Wahlprogrammen von ca. $70 \%$ auf ein relativ hohes Niveau von ungefähr $90 \%$ (T. Binder/A. M. Wüst 2004; A. M. Wüst/H. Schmitt 2007). ${ }^{1}$ Diese Zunahme geht nicht zulasten der nationalen Zuordnung, die relativ stabil zwischen 4 und 8\% liegt, sondern korrespondiert vielmehr mit einer starken Abnahme der unspezifischen Aussagen. Klar erkennbar ist also, dass die deutschen Parteien ihre Programme europäisch „verpacken“ (A. M. Wüst/D. Roth 2005: 61-62) und dass ,sich die deutschen Parteien mit ihren Manifestos darum [bemühen], dass Europawahlen nicht nur der Charakter von nationalen Nebenwahlen zugeschrieben wird.“(T. Binder/A. M. Wüst 2004: 40)

Das vorliegende Kapitel schließt sich dieser Forschung insofern nahtlos an, als auch hier die programmatischen Angebote der Parteien für die Europawahlen im Juni 2009 hinsichtlich ihres europäischen Framings untersucht werden. Dabei wird die Besonderheit berücksichtigt, dass den Europawahlen nur wenige Monate später eine Bundestagswahl folgte (September 2009). Darüber hinaus

1 Für 1979 wird ein europäisches Framing von 71\% ermittelt (T. Binder/A. M. Wüst 2004: 40), während für das Jahr 1999 unterschiedliche Ergebnisse vorliegen: 85\% (T. Binder/A. M. Wüst 2004: 40) bzw. 91,8\% (A. M. Wüst/H. Schmitt 2007: 80). 
liefert das Kapitel einen wichtigen Beitrag zur Weiterentwicklung der bisherigen Forschung. Dieser ist zum einen in der systematischen Konzeption des Nebenwahlcharakters auf der programmatischen Angebotsseite und zum anderen in der Verwendung eines modifizierten Kodierschemas zu sehen. Es wird zunächst theoretisch diskutiert, weshalb zu vermuten ist, dass die deutschen Parteien die Europawahlen als Nebenwahlen behandeln und anhand welcher Merkmale dieser Nebenwahlcharakter deutlich werden könnte. Anschließend erfolgt die empirische Überprüfung des Konzeptes, wobei im Gegensatz zur bisherigen Forschung das nationale bzw. europäische Framing von inhaltlichen Statements nur als ein Indikator aufgenommen wird.

\section{Theoretische Konzeption der Nebenwahlen auf der Angebotsseite}

Für Parteien ist prinzipiell jede Wahl relevant. In diesem Wettbewerb um politische Ideen können sie Mandate erringen, die ihnen einen größeren Einfluss auf politische Entscheidungsprozesse ermöglichen. Dies gilt auch für die Europawahlen, mit denen die Sitzverteilung im Europäischen Parlament festgelegt wird. In den letzten Jahren hat das Europäische Parlament zunehmend an Mitentscheidungskompetenzen gewonnen und ist damit aus der Sicht von Parteien als politikgestaltenden Akteuren von wachsender Relevanz. Deshalb mag es zunächst paradox erscheinen, die Programme für die Europawahlen auf ihren Nebenwahlcharakter hin zu untersuchen und nicht auf ihren genuinen Charakter als Bestandteil einer eigenständigen Wahl abzuzielen.

Werden Parteien jedoch als rational handelnde Akteure verstanden, die responsiv auf die Kontextbedingungen von Europawahlen reagieren und ihre knappen Ressourcen (Zeit, Geld, Personal) entsprechend einsetzen, dann lässt sich dieses Paradoxon auflösen. Als die zwei wichtigsten Kontextbedingungen für die Erstellung des programmatischen Angebots werden zum einen das Wahlverhalten bei Europawahlen und zum anderen die institutionellen Spezifika des Europäischen Parlaments berücksichtigt.

Drei Charakteristika des Wahlverhaltens bzw. der Nachfrageseite bei Nebenwahlen sind hier besonders hervorzuheben. Zunächst zeichnen sich Nebenwahlen durch ein geringeres Interesse seitens der Wähler aus, das sich in einer geringen Wahlbeteiligung niederschlägt. Zweitens werden die Wahlentscheidungen stark von der nationalen politischen Agenda beeinflusst, wobei drittens insbesondere kleinere und neue Parteien bei Europawahlen profitieren. Wie bereits oben dargelegt, spiegeln sich darin einerseits die wahren Parteipräferenzen wider, da keine Rücksicht auf eine Regierungsbildung genommen werden muss. Andererseits kann sich in der Wahl kleinerer und 
neuer Parteien ein Abstrafen der amtierenden Regierungsparteien ausdrücken, was den Nebenwahlcharakter besonders stark unterstreicht (K. Reif/H. Schmitt 1980).

Zudem stellen die institutionellen Besonderheiten des Europäischen Parlamentes relevante Kontextbedingungen für die Angebotserstellung der Parteien bei den Europawahlen dar. Mangels eines echten europäischen Parteienwettbewerbs müssen sich die nationalen Parteien dem Wettbewerb um die Verteilung der vertraglich zugesprochenen Sitze für ihren Mitgliedsstaat auf der nationalen Ebene stellen. Sie agieren demnach weniger als Europaparteien denn als nationale Parteien mit europäischem Profil. Damit kämpfen die Parteien sowohl bei den Hauptwahlen (Bundestagswahlen) als auch bei den Europawahlen in der nationalen Arena um Stimmen, bemühen sich also im Gegensatz zu anderen Nebenwahlen (z.B. Landtagswahlen) jeweils um Stimmen des gleichen Elektorats. Demzufolge liegt das Hauptaugenmerk der Parteien auf der nationalen Arena.

Des Weiteren relativieren sich die politischen Gestaltungsmöglichkeiten der nationalen Parteien im Europäischen Parlament, da die gewählten nationalen Delegierten dort nur eine (kleine) Gruppe in ihrer jeweiligen Fraktion bilden. Der Zusammenhang zwischen ihren Forderungen und der tatsächlich auf der EU-Ebene umgesetzten Politik ist daher sehr schwach ausgeprägt. Eng damit verknüpft ist der Umstand, dass die nationalen Parteien bei den Europawahlen keine Rücksicht auf potenzielle Koalitionspartner nehmen müssen. Zum einen geht aus dem Europäischen Parlament keine Regierung hervor. Zum anderen sind an der Mehrheitsbildung im Europäischen Parlament so viele andere Parteien beteiligt, dass es unmöglich ist, vorab im Europawahlkampf Koalitionsaussagen für das Agieren bei Abstimmungen im Europäischen Parlament zu treffen. Dies wird auch von den bisherigen Erfahrungen der Parteien gestützt, dass trotz der Tendenzen hinzu einer großen Koalition immer wieder Mehrheiten entsprechend der Politikinhalte gebildet werden (S. Hix 2001; A. Kreppel/S. Hix 2003; S. Hix et al. 2005).

Ungeachtet der gewachsenen Kompetenzen des Europäischen Parlamentes verdeutlichen diese Kontextbedingungen, dass die Anreize für die Parteien relativ hoch sind, Europawahlen weniger als eigenständige Wahlen als vielmehr als Nebenwahlen einzustufen und entsprechend $\mathrm{zu}$ agieren. Der Nebenwahlcharakter sollte sich demnach nicht nur in der Wahlkampfführung widerspiegeln (J. Tenscher 2005), sondern auch in der angebotenen Programmatik. 


\subsection{Indikatoren zur Messung des Nebenwahlcharakters}

Im Folgenden werden die drei Hauptmerkmale des Nebenwahlcharakters auf der Angebotsseite hergeleitet und Indikatoren für die Messung vorgestellt. Diese Hauptmerkmale leiten sich aus den skizzierten Kontextbedingungen und der Annahme ab, dass Parteien rational agieren und jene Bedingungen entsprechend antizipieren. Konkret ergeben sich daraus: erstens ein geringerer Ressourceneinsatz als bei Hauptwahlen, zweitens die Dominanz des nationalen Framings und drittens das Aufbrechen der nationalen Koalitionslogik.

(1) Der geringere Ressourceneinsatz ist einerseits auf das mangelnde Interesse an den Europawahlen auf der Nachfrageseite und andererseits auf die begrenzten Gestaltungsmöglichkeiten einer einzelnen Partei im Europäischen Parlament zurückzuführen. Da die Ressourcen von Parteien begrenzt sind und sie als rationale Akteure einen effektiven Mitteleinsatz anstreben, sollte sich die Angebotserstellung zwischen den nationalen Wahlen und den Europawahlen in dem Sinne unterscheiden, dass für Letztere weniger Ressourcen in die Programmentwicklung fließen. Ein erster Indikator kann hierbei in der Länge der Programme gesehen werden, wobei sich der Nebenwahleffekt in kürzeren Programmen zeigen sollte. Es ist davon auszugehen, dass der geringere Ressourceneinsatz zweitens dazu führt, dass eine geringere Anzahl von Parteiakteuren an der Ausarbeitung der Wahlprogramme beteiligt ist.

(2) Die Dominanz des nationalen Framings resultiert aus dem großen Einfluss der nationalen politischen Agenda auf das Wahlverhalten bei Europawahlen und aus den institutionellen Besonderheiten der Europawahlen, die einen nationalen Wettbewerb implizieren. Demzufolge zeigt sich der Nebenwahlcharakter, wenn Parteien in ihren Europawahlprogrammen stark auf das nationale Framing zurückgreifen und jeweils dieselben Themenbereiche mit der gleichen Relevanz bei den Haupt- und Nebenwahlen behandelt werden.

(3) Die verhältnismäßig große Anzahl von Wählern, die ihre Stimme bei Nebenwahlen für kleine und neuere Parteien abgeben und die fehlende Notwendigkeit, im Europäischen Parlament feste Koalitionen einzugehen und eine Regierung zu bilden, ermöglichen es den Parteien wiederum, ihre tatsächlichen Präferenzen in der Programmatik festzuhalten. Denn auf potenzielle Koalitionspartner muss keine Rücksicht genommen werden. In der Konsequenz sollte der Abstand zwischen den Parteien auf der Hauptkonfliktlinie, nämlich auf der Links-Rechts-Achse, bei Nebenwahlen wesentlich größer sein als bei Hauptwahlen.

Der Zusammenhang zwischen den Kontextbedingungen und dem Nebenwahlcharakter in der Programmatik wird abschließend in Tabelle 1 dargestellt. 
Festzuhalten ist: Je mehr dieser Merkmale zutreffen, desto stärker ist der Nebenwahlcharakter auf der Angebotsseite ausgeprägt.

\section{Tabelle 1: Merkmale und Indikatoren des Nebenwahlcharakters in der Programmatik}

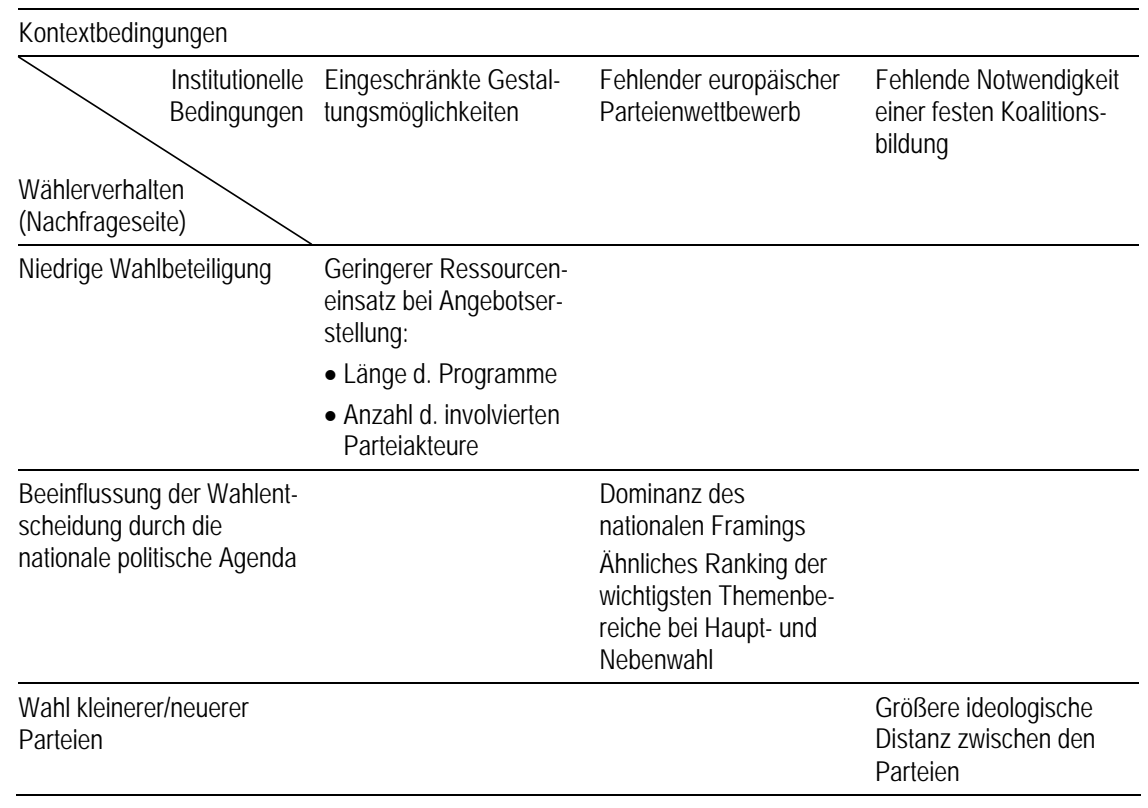

Allerdings stellt sich die Frage, weshalb das Ausmaß des Nebenwahlcharakters zwischen einzelnen Wahlprogrammen variieren kann. Die hier als relevant definierten Kontextbedingungen entlang der zwei Dimensionen Wählerverhalten und institutionelle Bedingungen des Europäischen Parlamentes bieten die Grundlage für einige Vermutungen hierzu. Zentral dabei ist, dass sich diese Kontextbedingungen in ihrer Relevanz für die einzelnen Parteien unterscheiden können. Im Rahmen der Analyse wird die Rolle der jeweiligen Partei im nationalen Parteienwettbewerb als besonderer Aspekt hervorgehoben.

Wie bereits oben diskutiert wurde, manifestiert sich die hohe Relevanz des nationalen Parteienwettbewerbs bei einer Nebenwahl in unterschiedlichen Facetten der Kontextbedingungen. Demzufolge sollte die Konstellation des nationalen Parteienwettbewerbs nicht ohne Rückwirkung auf das Ausmaß des Nebenwahlcharakters der Wahlprogrammatik bleiben. Konkret sind diesbezüglich 
Unterschiede zwischen den Regierungs- und Oppositionsparteien zu erwarten. Wenn Nebenwahlen den Zweck erfüllen, Regierungsparteien eine Rückmeldung zu ihren bisherigen Leistungen zu geben, und diese Parteien dabei in der Regel tatsächlich schlechter abschneiden, dann ist zu vermuten, dass sich Oppositionsparteien stärker auf nationale Themen bzw. nationales Framing konzentrieren. Dadurch können sie eine Unzufriedenheit der Wähler mit den Regierungsparteien für sich zu nutzen. Die Regierungsparteien wiederum werden sich stärker eines europäischen Framings bedienen, um einer Bewertung ihrer bisherigen Leistungen auf der nationalen Ebene zu entgehen. Mit anderen Worten: Auch wenn die Europawahlen sowohl für die Oppositions- als auch für die Regierungsparteien die Funktion von Nebenwahlen erfüllen, sollte die unterschiedliche Rationalität im Umgang mit dem nationalen Framing zu einem unterschiedlichen Ausmaß des Nebenwahlcharakters führen. Demzufolge lautet die weiterführende Untersuchungshypothese: Die Wahlprogramme der Oppositionsparteien weisen einen höheren Nebenwahlcharakter auf als die der Regierungsparteien.

\section{Empirische Analyse}

\subsection{Datenerhebung}

Um die Fragestellung nach Gemeinsamkeiten und Unterschieden in der angebotenen Parteiprogrammatik zwischen den Europa- und Bundestagswahlen 2009 hinreichend beantworten zu können, sind sowohl Daten zur Parteiorganisation als auch zur Programmatik notwendig. Angaben über involvierte Parteiakteure wurden den Satzungen sowie relevanten Beschlüssen der Parteien entnommen und um Erkenntnisse aus dem EU-Profiler-Projekt ${ }^{2}$ und aus der Sekundärliteratur ergänzt.

Die empirische Analyse des programmatischen Angebots basiert auf einer systematischen inhaltsanalytischen Auswertung der Europa- und Bundestagswahlprogramme der Parteien. Analysiert wurden die Programme der im deutschen Parteienwettbewerb von 2009 relevanten Parteien: Bündnis 90/ Die Grünen, CDU, CSU, DIE LINKE, FDP und SPD. Während CDU und CSU bei den Bundestagswahlen als ein Akteur betrachtet werden, erfolgt eine separate Analyse der Programme zur Europawahl, da sie dort unabhängig voneinander und mit jeweils eigenem Programm antraten.

2 Der „EU Profiler“ ist eine europaweite Online-Wahlhilfe zur Europawahl 2009. Mehr unter http://www.euprofiler.eu und A. H. Trechsel/P. Mair 2009. 
Die inhaltsanalytische Auswertung beruht auf dem Verfahren und dem Kodierschema des Comparative-Manifesto-Projekts (CMP) (I. Budge et al. 1987; I. Budge et al. 2001) und folgt den Anweisungen des entsprechenden Handbuchs für die CMP-Kodierung (A. Volkens 2002). ${ }^{3}$ Im Gegensatz zum bereits erwähnten „Euromanifesto Project“ (A. M. Wüst/A. Volkens 2003) wurde das grundlegende CMP-Kodierschema nicht verändert, sondern lediglich um zwei Codes ergänzt. Als erstes wurde ein weiterer Europacode eingeführt, der Positionen zur europäischen Integration differenzierter als die bekannten Codes für Aussagen pro und contra EU-Integration erfassen kann. Konkret wurden damit jene Positionen kodiert, die sich zwischen einer uneingeschränkten pro-europäischen Haltung und einer klaren Verneinung der EU-Integration einordnen lassen. Die zweite Ergänzung besteht in der Einführung eines Codes für negative Äußerungen zur Landwirtschaft, die beispielsweise in der Ablehnung einer weiteren Subventionierung deutlich werden.

Des Weiteren wurde die Idee des „Euromanifesto Projects“, von Parteien benannte Regierungsebenen gesondert mittels sogenannter Ebenencodes zu erfassen, aufgenommen und weiterentwickelt. Als Ergebnis wurde jeder QuasiSatz mit einem CMP-Code und einem zweistelligen Ebenencode versehen. Der erste Teil des Ebenencodes bildet ab, welche Ebene von den Parteien angesprochen bzw. als handelnde Ebene postuliert wird. Hierbei wird zwischen neun Ebenen unterschieden (lokal, regional, national, europäisch, international sowie zwischen verschiedenen Konstellationen mit Deutschland als zentralem Akteur). Der zweite Teil des Ebenencodes erfasst die benannte Kompetenzverteilung zwischen den Ebenen und spiegelt die normative Bewertung der Parteien wider. Drei Konstellationen sind dabei möglich: (1) Es wird keine Veränderung gefordert, (2) die angesprochene Ebene soll weniger Kompetenzen erhalten bzw. in geringerem Umfang verbindliche Entscheidungen treffen oder (3) der Ebene sollen mehr Kompetenzen zugesprochen werden bzw. sie soll aktiver an der Entscheidungsfindung respektive Politikumsetzung teilnehmen. Außerdem können die Einstellungen der Parteien durch spezielle Codes z. B. zum Subsidiaritätsprinzip, zur Zusammenarbeit mehrerer Ebenen und zum Lissabonner Vertrag abgebildet werden.

3 Die Autorinnen kodierten die Europawahlprogramme selbst. Die Reliabilität dieser Datenerhebung wurde durch die Kodierung in mehreren Runden und mehrere Kodierbesprechungen hergestellt. Die Daten der Bundestagswahlprogramme wurden dem Comparative-Manifesto-Projekt (CMP) entnommen. 


\subsection{Ressourceneinsatz für die Angebotserstellung}

Prinzipiell setzt das deutsche Parteiengesetz recht enge Grenzen für den organisatorischen und funktionellen Aufbau von Parteien, die sich in den Satzungen bzw. Statuten der Parteien wiederfinden. Hinsichtlich der programmatischen Ausrichtung ist im deutschen Parteiengesetz allerdings nur festgehalten, dass Parteien ein Programm benötigen ( $\$ 6$ Abs. 1), das von den Parteitagen zu beschließen ist ( 9 Abs. 3). Da Parteiprogramme eher den Grundsatzprogrammen entsprechen als den (spezialisierten) Wahlprogrammen, müssen die deutschen Parteien weder bei der Entwicklung noch beim Beschluss von Wahlprogrammen gesetzliche Vorgaben berücksichtigen.

Die Analyse von Wahlprogrammen beruht auf der grundlegenden Annahme, diese würden die Positionen der Parteien am besten widerspiegeln, da die sie einer Zustimmung durch den Parteitag bedürfen und deshalb als allgemein geteilte Positionen der Gesamtpartei interpretiert werden können (I. Budge 2001). In der Parteienforschung ist bislang jedoch nur rudimentär beleuchtet worden, wie Wahlprogramme innerhalb von Parteien entstehen. Dies ist insofern überraschend, als verantwortlichen Akteuren eine besondere Agenda-Setter-Funktion zugeschrieben werden kann und sie damit entscheidend dazu beitragen, welche Themen und Positionen in der Programmatik aufgegriffen werden. Dieses Kapitel kann diese Forschungslücke nicht schließen, wird aber einzelne Aspekte hervorheben, die zur Bewertung des Ressourceneinsatzes für die programmatische Angebotserstellung bei Bundestags- und Europawahlen relevant sind.

Den Satzungen der Parteien kann nur indirekt entnommen werden, welches Parteigremium für den Entwurf von Wahlprogrammen zuständig ist. In der Regel werden dort nur Beratungen grundsätzlicher und organisatorischer Aufgaben erwähnt, die in den Parteien unterschiedlichen Organisationseinheiten wie etwa dem Bundesausschuss (CDU, DIE LINKE), dem Parteiausschuss (CSU), dem Parteirat (SPD, Bündnis 90/Die Grünen) oder dem Bundespräsidium (FDP) zugewiesen werden. Dies lässt viel Raum für Ad-hoc-Konstellationen ( $T$. Poguntke 2007). ${ }^{4}$ Die Entwürfe von Wahlprogrammen entstehen somit in starker Abhängigkeit von Persönlichkeiten und Netzwerkstrukturen innerhalb der Parteien. Diese Konstellationen können nicht nur zwischen den Europa- und Bundestagswahlen, sondern auch von Wahl zu Wahl variieren. Ein kleiner Unterschied ist dahingehend feststellbar, dass bei den Europawahlen verstärkt EU-

4 Die empirischen Erkenntnisse beruhen im Wesentlichen auf 24 Eliteninterviews mit Repräsentanten deutscher Parteien (T. Poguntke 2007: 130). 
Spezialisten in den Programmentwurf einbezogen werden, worunter Abgeordnete des Europäischen Parlamentes und Spezialisten der Bundestagsfraktionen fallen (T. Poguntke 2007: 122).

Auch der Weg vom ersten Entwurf zum endgültigen Beschluss ist bei den Europa- und Bundestagswahlen sowohl innerhalb der als auch zwischen den Parteien recht uneinheitlich. So diskutieren die jeweiligen Regionalkonferenzen von Bündnis 90/Die Grünen, der FDP und der Linken sowohl die Entwürfe für die Bundestags- als auch für die Europawahlen. Bei der SPD lässt sich nur eine Beteiligung ihrer Regionalkonferenzen bei der Bundestagswahl erkennen. Für die CDU wiederum ist nicht bekannt, ob neben dem Bundesvorstand, der das alleinige Beschlussorgan ist, andere Parteigremien in die Ausarbeitung der Wahlprogramme involviert sind. Der „Europakongress“ am 16. März 2009 konnte keinen Einfluss auf die Programmatik nehmen, da es sich lediglich um eine Podiumsdiskussion handelte. Ähnliches ist für die CSU festzuhalten. Auch wenn ihre Mitglieder vor der Europawahl aufgefordert waren, über das Internet Themen für das Europawahlprogramm vorzuschlagen, blieb die Programmentwicklung dem jeweiligen Beschlussorgan vorbehalten.

Welches Parteiorgan Wahlprogramme endgültig verabschiedet, spiegelt nicht nur das partizipatorische Grundverständnis einer Partei wider, sondern auch welche Ressourcen für die Bundestags- und Europawahlen bereitgestellt werden (siehe Tabelle 2). Abgesehen von CDU und CSU ist bei allen Parteien ein Parteitag das endgültige Beschlussgremium. Unabhängig davon, ob es sich um ein Bundestags- oder ein Europawahlprogramm handelt, bleiben die Beschlussgremien bei den Grünen, bei der FPD, der CDU sowie bei den Linken dieselben.

In der CSU hingegen werden für den Beschluss über das Europawahlprogramm mehr Funktionsträger einbezogen, da der Parteiausschuss neben dem Parteivorstand weitere parteiinterne Akteure umfasst. $^{5}$ Für die Sozialdemokraten kann ein geringerer Ressourceneinsatz beim Beschluss des Europawahlprogramms konstatiert werden, da die eingesetzte Delegiertenkonferenz wesentlich kleiner ist als beim Bundeswahlprogramm. ${ }^{6}$

5 So gehören dem CSU-Parteiausschuss auch die Bezirksvorsitzenden, die Delegierten der Bezirksverbände, ausgewählte VertreterInnen des Europäischen Parlamentes, des Deutschen Bundestages sowie des Bayerischen Landtages und die Landesvorsitzenden der Arbeitsgemeinschaften an.

6 Darüber hinaus zeigen erste vorläufige Forschungsergebnisse zu den Wahlprogrammen der Europaparteien 2009, dass Parteien Synergieeffekte nutzen: Am Beispiel der Europäischen Volkspartei (EVP) und insbesondere der Europäischen Sozialdemokraten (PES) kann bereits gezeigt werden, dass nationale Parteivertreter die europäischen Manifestos mitentwickeln und wiederum Teile der dortigen Programmatik für die nationalen Wahlen übernehmen. Die SPD 
Hinsichtlich der involvierten parteiinternen Akteure lassen sich also nach bisherigen Kenntnissen nur bei den Sozialdemokraten Nebenwahleffekte konstatieren, da dort bei den Europawahlen sowohl an der Programmentwicklung als auch an der Beschlussfassung weniger Akteure beteiligt sind.

Tabelle 2: Beschlussorgane der Europa- und Bundestagswahlprogramme

\begin{tabular}{|l|c|c|}
\hline & $\begin{array}{c}\text { Beschlussorgan des } \\
\text { Europawahlprogramms }\end{array}$ & $\begin{array}{c}\text { Beschlussorgan des } \\
\text { Bundestagswahlprogramms }\end{array}$ \\
\hline CDU & Bundesvorstand (16. März 2009) & $\begin{array}{c}\text { Bundesvorstand der CDU (28. Juni 2009); } \\
\text { gemeinsames Programm mit der CSU }\end{array}$ \\
\hline CSU & Parteiausschuss (9. Mai 2009) & $\begin{array}{c}\text { Parteivorstand CSU (28. Juni 2009); } \\
\text { gemeinsames Programm mit der CDU }\end{array}$ \\
\hline SPD & $\begin{array}{c}\text { Europadelegiertenkonferenz } \\
\text { (8. Dezember 2008); 280 Delegierte }\end{array}$ & $\begin{array}{c}\text { Außerordentlicher Parteitag } \\
\text { (14. Juni 2009); 480 Delegierte }\end{array}$ \\
\hline FDP & $\begin{array}{c}\text { Europaparteitag (7. Januar 2009); } \\
\text { 662 Delegierte }\end{array}$ & $\begin{array}{c}\text { Bundesparteitag (15.-17. Mai 2009); } \\
\text { 662 Delegierte }\end{array}$ \\
\hline Die Grünen & $\begin{array}{c}\text { Bundesdelegiertenkonferenz } \\
\text { (23.-25. Januar 2009); 840 Delegierte }\end{array}$ & $\begin{array}{c}\text { Bundesdelegiertenkonferenz } \\
\text { (8.-10. Mai 2009); 840 Delegierte }\end{array}$ \\
\hline DIE LINKE & $\begin{array}{c}\text { Europaparteitag (28. Februar 2009); } \\
\text { 562 Delegierte, ein Mandat unbesetzt }\end{array}$ & $\begin{array}{c}\text { Bundestagswahlparteitag } \\
\text { (20.-21. Juni 2009); }\end{array}$ \\
\begin{tabular}{c} 
562 Delegierte, ein Mandat unbesetzt \\
\hline
\end{tabular}
\end{tabular}

Quelle: Eigene Darstellung nach Angaben der deutschen Parteien.

Auch die Länge von Wahlprogrammen kann als ein Indikator für den Ressourceneinsatz dienen. Wie oben dargelegt, erfordert das Verfassen längerer Programme mehr Personal-, Expertise- und Zeitressourcen. Wird die Anzahl der Kodiereinheiten (Quasi-Sätze) als Grundlage des Vergleichs verwendet, dann zeigt sich eindeutig ein klarer Nebenwahlcharakter für die Europawahlpro-

übernahm nach derzeitigem Stand der Auswertung ca. 30\% der Argumente des Euro-Manifestos und weist damit die mit Abstand höchste Übereinstimmung zwischen beiden Programmen auf. Bei der CDU treffen ca. $13 \%$ der Aussagen überein, was vergleichsweise wenig ist, aber innerhalb der EVP-Familie dennoch einen relativ hohen Wert darstellt (M. Sigalas/M. Mokre 2010: 20-21). 
gramme aller Parteien: Sämtliche Europawahlprogramme sind signifikant kürzer (siehe Tabelle 3).

\section{Tabelle 3: Länge der Europa- und Bundestagswahlprogramme}

\begin{tabular}{lcc}
\hline Partei & Europawahl 2009 & Bundestagswahl 2009 \\
\hline CDU/CSU & - & 2008 \\
\hline CDU & 341 & - \\
\hline CSU & 321 & - \\
\hline SPD & 459 & 2208 \\
\hline FDP & 640 & 2250 \\
\hline Bündnis 90/Die Grünen & 2018 & 3596 \\
\hline DIE LINKE & 833 & 1666
\end{tabular}

Quelle: Eigene Berechnung. Absolute Zahlen der Quasi-Sätze.

\subsection{Inhaltsanalyse der Wahlprogramme}

\subsubsection{Framing der Europawahlprogramme}

Die Ergebnisse zum Framing in früheren Europawahlprogrammen lassen sich für das Jahr 2009 nur teilweise bestätigen. Generell „,verpackten“ die deutschen Parteien ihre Programme auch bei der jüngsten Europawahl in ein europäisches Framing. Die hohen Anteilswerte der vorangegangenen Wahlen werden jedoch nicht erreicht. ${ }^{7}$ Zwischen knapp 54\% (CSU) und etwas über 67\% (SPD) aller programmatischen Statements sind mit einem eindeutigen europäischen Bezug versehen (siehe Tabelle 4). Die zusätzliche Berücksichtigung der subnationalen Ebenen in der Kodierung kann eine starke Positionierung der CSU als regionale Partei verdeutlichen, wohingegen der CDU der höchste Anteil eines nationalen Framings (13,7\%) zuzuschreiben ist. Gerade vor dem Hintergrund der internationalen Finanzkrise und des Klimawandels wurden darüber hinaus vielfach Forderungen nach internationalen Lösungen geäußert. Diese wurden am stärksten von den Grünen (11,6\%) und am wenigsten von den Liberalen $(1,6 \%)$ vertreten. Insgesamt kann für keine der deutschen Parteien eine eindeutige Domi-

7 Auffallend ist hier im Vergleich der relativ hohe Anteil von Statements, die keinem spezifischen Framing zugeordnet werden können. 
nanz des nationalen Framings festgestellt werden. Mit anderen Worten: Dieses Nebenwahlmerkmal wird von keiner Partei erfüllt.

Tabelle 4: Verwendung von Framings in den Europawahlprogrammen

\begin{tabular}{|c|c|c|c|c|c|c|}
\hline Parteien & CDU & CSU & SPD & FDP & $\begin{array}{l}\text { DIE } \\
\text { LINKE }\end{array}$ & $\begin{array}{l}\text { Bündnis } \\
\text { 90/Die } \\
\text { Grünen }\end{array}$ \\
\hline lokal & 1,8 & 1,9 & 0,4 & 0,3 & 1,0 & 2,8 \\
\hline regional & 0,0 & 13,1 & 0,0 & 0,0 & 0,8 & 2,1 \\
\hline subnational (lokal + regional) & 1,8 & 15,0 & 0,4 & 0,3 & 1,8 & 4,9 \\
\hline national & 12,9 & 10,0 & 7,2 & 9,8 & 5,8 & 7,7 \\
\hline $\begin{array}{l}\text { gesamt national } \\
\text { (subnational + national) }\end{array}$ & 14,7 & 25,0 & 7,6 & 10,1 & 7,6 & 12,6 \\
\hline EU & 66,4 & 53,6 & 67,1 & 61,6 & 64,5 & 58,6 \\
\hline international & 9,1 & 2,8 & 6,3 & 1,6 & 3,8 & 11,6 \\
\hline keine eindeutige Zuordnung & 7,3 & 12,2 & 11,8 & 23,6 & 22,2 & 15,0 \\
\hline
\end{tabular}

Angaben: Anteil an Quasi-Sätzen in Prozent, Rundung auf die erste Stelle nach dem Komma.

\subsubsection{Ranking der Themen}

Werden Themen häufiger als andere erwähnt, dann unterstreichen Parteien damit eine besondere Relevanz dieser Problembereiche. Der Nebenwahlcharakter zeichnet sich dadurch aus, dass trotz unterschiedlicher Kompetenzen der europäischen und der Bundesebene jene Themenbereiche die Europawahlprogramme dominieren, die auch bei der Bundestagswahl am wichtigsten sind.

Mittels der Messung von Häufigkeiten kann eine Rangordnung der Themen ermittelt werden, wobei die jeweilige Positionierung dafür unerheblich ist (siehe Tabelle 5). Die prozentualen Anteile der Quasi-Sätze diverser Themenbereiche werden berechnet und die zehn wichtigsten in die Analyse einbezogen. Um eine gewisse Kontinuität zu den Analysen früherer Europawahlen zu gewährleisten, sind die Themenbereiche hier an A. M. Wüst (2009) angelehnt. Der Themenbereich Europäische Union bleibt in der vorliegenden Auswertung allerdings un- 
berücksichtigt, da aufgrund der Verwendung der neuen Ebenencodes die EUCodes des CMP-Kodierschemas an Bedeutung verlieren.

Als Erstes zeigt eine Überprüfung der thematischen Übereinstimmung zwischen den Europa- und Bundestagswahlen, dass durchaus unterschiedliche Themenbereiche angesprochen werden. So werden in den Europawahlprogrammen der Grünen, der Sozialdemokraten, der Linken und der Liberalen jeweils zwei Themenbereiche aufgegriffen, die nicht Bestandteil der Bundestagswahlprogramme sind. Bei den Liberalen handelt es sich um „Demokratie“ und „Landwirtschaft", denen eine mittlere Relevanz (gemessen an der Rangordnung) im Europawahlprogramm eingeräumt wird und die quasi gegen weniger relevante Aspekte im Bundeswahlprogramm eingetauscht wurden. Die abweichenden Themenbereiche in den Europawahlprogrammen der drei anderen Parteien (Grüne, Linke, SPD) sind von geringerer Bedeutung, da fast alle auf den hinteren Plätzen der Rangordnung zu finden sind und jeweils einen Anteil zwischen 2 und $4 \%$ am Gesamtprogramm ausmachen. Die größten Abweichungen sind bei der CDU und der CSU festzustellen: Vier andere Themenbereiche sind im mittleren bis hinteren Bereich in der Rangordnung ihres Europawahlprogramms zu finden. Das deutet nicht nur auf einen geringeren Nebenwahleffekt hin, sondern unterstreicht auch die durchaus abweichenden Ansichten beider Parteien, die im gemeinsamen Bundestagswahlprogramm nicht deutlich werden.

Zusätzlich können die Rangordnungen dahingehend analysiert werden, inwieweit denselben Themenbereichen die gleiche Bedeutung zugeschrieben wird. Geringe Abweichungen liegen dann vor, wenn die Positionen in der Rangordnung gleich sind bzw. nur um maximal zwei Positionen abweichen. Als große Abweichungen werden jene Veränderungen in der Rangordnung interpretiert, die mehr als zwei Positionen umfassen. Auf dieser Basis lässt sich konstatieren, dass die Wahlprogramme der Sozialdemokraten mit großem Abstand zu den anderen Parteien die geringsten thematischen Abweichungen aufweisen. Auch die Programme der Grünen stimmen relativ stark überein. Eine stärkere Variation ist für die FDP und die CSU festzuhalten, wohingegen die größten Abweichungen bei der Linken und der CDU festgestellt werden können.

Werden jene Themen näher beleuchtet, die nur in den Europawahlprogrammen zu den zehn wichtigsten gehören, dann zeigt sich ein expliziter EU-Bezug bei der FDP, CSU und CDU: Es wird der Bereich „Landwirtschaft“ thematisiert und damit der dominierenden europäischen Kompetenz in diesem Politikfeld Rechnung getragen. Des Weiteren greifen CDU und CSU den Bereich „Nation“ auf, der sich explizit aus dem Kontext der Europawahlen ergibt, da vor dem europäischen Hintergrund die eigene Nation herausgestellt wird, was im Bun- 
destagswahlprogramm in dieser Form nicht relevant ist. Demzufolge ist hier der Nebenwahlcharakter abgeschwächt.

Zusammengefasst zeigt dieser Indikator eine unterschiedliche Ausprägung und deutet auf ein differenziertes Ausmaß des Nebenwahlcharakters je nach Partei. Während dieses Merkmal für die SPD eindeutig zutrifft, kann es der CDU und der CSU eindeutig abgesprochen werden. Eine schwache Ausprägung ist für die Grünen, die Liberalen und DIE LINKE feststellbar. 
Tabelle 5: Themenranking im Vergleich

\begin{tabular}{|c|c|c|c|c|c|c|c|c|c|c|c|c|}
\hline \multirow[b]{2}{*}{ Rang } & \multicolumn{2}{|c|}{ CDU } & \multicolumn{2}{|c|}{ CSU } & \multicolumn{2}{|c|}{ SPD } & \multicolumn{2}{|c|}{ FDP } & \multicolumn{2}{|c|}{ DIE LINKE } & \multicolumn{2}{|c|}{ Bündnis 90/Die Grünen } \\
\hline & BTW09 & EPW09 & BTVO9 & EPMO9 & BTVO9 & EPMO9 & BTVO9 & EPMO9 & BTVO9 & EPMO9 & BTVO9 & EPMO9 \\
\hline 1 & Wirtschaft & Wirtschaft & Wirtschaft & Wirtschaft & Wirtschaft & Wirtschaft & Wirtschaft & Wirtschaft & Wohlfahrt & Wrtschaft & Wirtschaft & Umwelt \\
\hline 2 & $\begin{array}{l}\text { Politisches } \\
\text { System }\end{array}$ & Außenpolitik & $\begin{array}{c}\text { Politisches } \\
\text { System }\end{array}$ & $\begin{array}{c}\text { Politisches } \\
\text { System }\end{array}$ & Wohlfahrt & Wohlfahrt & $\begin{array}{c}\text { Politisches } \\
\text { System }\end{array}$ & $\begin{array}{c}\text { Politisches } \\
\text { System }\end{array}$ & Wrtschaft & Außenpolitik & Wohlfahrt & Wrtschaft \\
\hline 3 & Wohlfahrt & Sicherheit & Wohlfahrt & Demokratie & Arbeitnehmer & - Arbeitnehmer & Wohlfahrt & Umwelt & $\begin{array}{l}\text { Soziale } \\
\text { Gruppen }\end{array}$ & Umwelt & Umwelt & Wohlfahrt \\
\hline 4 & Sicherheit & $\begin{array}{l}\text { Menschen- } \\
\text { rechte }\end{array}$ & Sicherheit & Die Nation & Umwelt & Außenpolitik & $\begin{array}{l}\text { Menschen- } \\
\text { rechte }\end{array}$ & Demokratie & Außenpolitik & Wohlfahrt & $\begin{array}{l}\text { Soziale } \\
\text { Gruppen }\end{array}$ & $\begin{array}{l}\text { Menschen- } \\
\text { rechte }\end{array}$ \\
\hline 5 & Umwelt & Die Nation & Umwelt & Landwirtschaft & Außenpolitik & $\begin{array}{l}\text { Menschen- } \\
\text { rechte }\end{array}$ & Bildung & Landwirtschaft & Unmelt & $\begin{array}{l}\text { Menschen- } \\
\text { rechte }\end{array}$ & $\begin{array}{l}\text { Menschen- } \\
\text { rechte }\end{array}$ & Außenpolitik \\
\hline 6 & $\begin{array}{l}\text { Soziale } \\
\text { Gruppen }\end{array}$ & $\begin{array}{c}\text { Politisches } \\
\text { System }\end{array}$ & $\begin{array}{l}\text { Soziale } \\
\text { Gruppen }\end{array}$ & Außenpolitik & Bildung & Umwelt & $\begin{array}{l}\text { Soziale } \\
\text { Gruppen }\end{array}$ & $\begin{array}{l}\text { Menschen- } \\
\text { rechte }\end{array}$ & Demokratie & Sicherheit & $\begin{array}{c}\text { Politisches } \\
\text { System }\end{array}$ & $\begin{array}{l}\text { Politisches } \\
\text { System }\end{array}$ \\
\hline 7 & Außenpolitik & $\begin{array}{l}\text { Politische } \\
\text { Autorität }\end{array}$ & Außenpolitik & Arbeitnehmer & Sicherheit & Sicherheit & Außenpolitik & Außenpolitik & Arbeitnehmer & Demokratie & Außenpolitik & Demokratie \\
\hline 8 & Werte & LandMirtschaft & Werte & Umwelt & $\begin{array}{l}\text { Soziale } \\
\text { Gruppen }\end{array}$ & $\begin{array}{l}\text { Soziale } \\
\text { Gruppen }\end{array}$ & Umwelt & Sicherheit & $\begin{array}{l}\text { Politische } \\
\text { Autorität }\end{array}$ & $\begin{array}{l}\text { Soziale } \\
\text { Gruppen }\end{array}$ & $\begin{array}{l}\text { Politische } \\
\text { Autorität }\end{array}$ & Arbeitnehmer \\
\hline 9 & Kultur/Sport & Umwelt & Kultur/ Sport & Sicherheit & $\begin{array}{l}\text { Politisches } \\
\text { System }\end{array}$ & $\begin{array}{c}\text { Politisches } \\
\text { System }\end{array}$ & Sicherheit & Bildung & $\begin{array}{l}\text { Menschen- } \\
\text { rechte }\end{array}$ & Bildung & Demokratie & Sicherheit \\
\hline 10 & $\begin{array}{c}\text { Z゙vil- } \\
\text { gesellschaft }\end{array}$ & Wohlfahrt & $\begin{array}{c}\text { Z vil- } \\
\text { gesellschaft }\end{array}$ & Werte & Demokratie & $\begin{array}{c}\text { Politische } \\
\text { Autorität }\end{array}$ & Kultur/Sport & Wohlfahrt & Sicherheit & $\begin{array}{c}\text { Politisches } \\
\text { System }\end{array}$ & Bildung & Bildung \\
\hline
\end{tabular}

Angaben: Ranking anhand der prozentualen Anteile (hier nicht abgebildet) ermittelt. BTW 09: Bundestagswahlprogramm 2009; EPW 09: Europawahlprogramm 2009. 


\subsubsection{Ideologische Distanz zwischen den Parteien}

Wie oben dargelegt unterscheiden sich die Europa- von den Bundestagswahlen dahingehend, dass die Parteien bei den Ersteren keine Rücksicht auf potenzielle Koalitionspartner nehmen müssen und deshalb ihre Positionen wesentlich dezidierter artikulieren können. Dementsprechend äußert sich der Nebenwahleffekt in einer größeren Distanz der Parteien auf der Links-Rechts-Achse. Die Berechnung der Parteipositionen auf dieser Achse erfolgt im Wesentlichen nach dem etablierten CMP-Prinzip (M. Laver/I. Budge 1992). ${ }^{8}$ Dabei wird der prozentuale Anteil linker Kategorien an der Gesamtzahl der Quasi-Sätze vom Anteil der rechten Kategorien abgezogen. Der Wertebereich ist daher zwischen -100 und +100 definiert, wobei ein kleinerer Wert eine Parteiposition indiziert, die weiter links liegt.

Da für den hier zu analysierenden Indikator weniger die Positionen der Parteien auf der Links-Rechts-Achse als vielmehr der Abstand zwischen ihnen relevant ist, wurde die Distanz jeder Partei zu den jeweils anderen bei den Europa- und den Bundestagswahlen berechnet (siehe Tabelle 6). Im Ergebnis zeigt sich ein sehr klares Muster: Bis auf kleine Ausnahmen vergrößert sich auf der europäischen Ebene der Abstand jeder Partei zu den jeweils anderen Parteien. ${ }^{9}$ Dies trifft nicht nur auf jene Parteien zu, zwischen denen die ideologische Distanz bekanntermaßen sehr groß ist, z. B. zwischen der Linken und der CDU bzw. CSU. Bei den Europawahlen ist auch gerade zwischen jenen Parteien eine größere ideologische Distanz festzustellen, die auf der Bundesebene potenziell als Koalitionspartner betrachtet werden - sei es, weil sich diese Parteien zum Zeitpunkt des Entstehens der Europawahlprogramme in einer Koalitionsregierung befanden oder weil sie in der Vergangenheit eine Koalition gebildet hatten. Demzufolge kann der Nebenwahleffekt einer größeren Distanz auf der LinksRechts-Achse für alle Parteiprogramme attestiert werden.

8 Abweichend wird der zur Linkskodierung gehörende Code 202 (Demokratie) nicht in die Berechnung der Position einbezogen, da dessen Berücksichtigung bei den Europawahlprogrammen zu einer unverhältnismäßig starken Verschiebung der Parteipositionen nach links führt. Hintergrund ist, dass die Parteien mehr oder weniger ausgeprägt auf das Demokratiedefizit in der EU eingehen. Da es sich bei der Perzeption von Demokratie nicht um ein genuin „linkes“ Thema handelt, würde demzufolge die Berücksichtigung dieses Codes die Vergleichbarkeit der Links-rechts-Position bei Bundestags- und Europawahlen stark einschränken.

9 Die FDP weicht von diesem Muster etwas ab, da sich ihre Distanz zu den Grünen und zur Linken im Europawahlprogramm etwas verringert. Ursächlich hierfür ist der verhältnismäßig geringe Anteil an wirtschaftlichen Kategorien, die wiederum in die Rechtspositionierung einfließen. 
Tabelle 6: Distanz zwischen den Parteien auf der Links-Rechts-Achse

\begin{tabular}{|c|c|c|c|c|c|c|}
\hline & CDU & CSU & SPD & FDP & DIE LINKE & $\begin{array}{l}\text { Bündnis 90/ } \\
\text { Die Grünen }\end{array}$ \\
\hline CDU & - & $\begin{array}{c}\text { BTW: } 0 \\
\text { EPW: } 2,1\end{array}$ & $\begin{array}{l}\text { BTW: } 24,7 \\
\text { EPW: } 37,2\end{array}$ & $\begin{array}{l}\text { BTW: } 3,9 \\
\text { EPW: } 7,4\end{array}$ & $\begin{array}{l}\text { BTW: } 28,9 \\
\text { EPW: } 30,3\end{array}$ & $\begin{array}{l}\text { BTW: } 19,1 \\
\text { EPW: } 20,0\end{array}$ \\
\hline CSU & $\begin{array}{c}\text { BTW: } 0 \\
\text { EPW: } 2,1\end{array}$ & - & $\begin{array}{l}\text { BTW: } 24,7 \\
\text { EPW: } 39,4\end{array}$ & $\begin{array}{l}\text { BTW: } 3,9 \\
\text { EPW: } 9,5\end{array}$ & $\begin{array}{l}\text { BTW: } 28,9 \\
\text { EPW: } 32,4\end{array}$ & $\begin{array}{l}\text { BTW: } 19,1 \\
\text { EPW: } 22,2\end{array}$ \\
\hline SPD & $\begin{array}{l}\text { BTW: } 24,7 \\
\text { EPW: } 37,2\end{array}$ & $\begin{array}{l}\text { BTW: } 24,7 \\
\text { EPW: } 39,4\end{array}$ & - & $\begin{array}{l}\text { BTW: } 20,8 \\
\text { EPW: } 29,9\end{array}$ & $\begin{array}{l}\text { BTW: } 4,2 \\
\text { EPW: } 7,0\end{array}$ & $\begin{array}{c}\text { BTW: } 5,5 \\
\text { EPW: } 17,2\end{array}$ \\
\hline FDP & $\begin{array}{l}\text { BTW: } 3,9 \\
\text { EPW: } 7,4\end{array}$ & $\begin{array}{l}\text { BTW: } 3,9 \\
\text { EPW: } 9,5\end{array}$ & $\begin{array}{l}\text { BTW: } 20,8 \\
\text { EPW: } 29,9\end{array}$ & - & $\begin{array}{l}\text { BTW: } 25,0 \\
\text { EPW: } 22,9\end{array}$ & $\begin{array}{l}\text { BTW: } 15,3 \\
\text { EPW: } 12,7\end{array}$ \\
\hline $\begin{array}{l}\text { DIE } \\
\text { LINKE }\end{array}$ & $\begin{array}{l}\text { BTW: } 28,9 \\
\text { EPW: } 30,3\end{array}$ & $\begin{array}{l}\text { BTW: } 28,9 \\
\text { EPW: } 32,4\end{array}$ & $\begin{array}{l}\text { BTW: } 4,2 \\
\text { EPW: } 7,0\end{array}$ & $\begin{array}{l}\text { BTW: } 25,0 \\
\text { EPW: } 22,9\end{array}$ & - & $\begin{array}{l}\text { BTW: } 9,8 \\
\text { EPW: } 10,2\end{array}$ \\
\hline $\begin{array}{l}\text { Bündnis 90/ } \\
\text { Die Grünen }\end{array}$ & $\begin{array}{l}\text { BTW: } 19,1 \\
\text { EPW: } 20,0\end{array}$ & $\begin{array}{l}\text { BTW: } 19,1 \\
\text { EPW: } 22,2\end{array}$ & $\begin{array}{c}\text { BTW: } 5,5 \\
\text { EPW: } 17,2\end{array}$ & $\begin{array}{l}\text { BTW: } 15,3 \\
\text { EPW: } 12,7\end{array}$ & $\begin{array}{c}\text { BTW: } 9,8 \\
\text { EPW: } 10,2\end{array}$ & - \\
\hline
\end{tabular}

Angaben: Differenzen zwischen den Positionen, gerundet auf eine Stelle nach dem Komma; BTW: Bundestagwahlprogramm, EPW: Europawahlprogramm; Hervorhebung: potenzielle Koalitionspartner.

\subsection{Europawahlen: Auch Nebenwahlen auf der Angebotsseite?}

Die Untersuchung des Nebenwahlcharakters anhand mehrerer Merkmale zeigt, dass zum einen das Ressourcenmerkmal in Teilen für alle Parteien zutrifft und zum anderen die ideologische Distanz zwischen allen Parteien auf der europäischen Ebene größer ist. Doch die Inhaltsanalyse verdeutlicht die Notwendigkeit einer weitaus differenzierteren Betrachtung der Angebotsseite hinsichtlich ihres potenziellen Nebenwahlcharakters. Es zeigt sich, dass keine Dominanz des nationalen Framings festgestellt werden kann und demnach die Europawahlen von den Parteien dezidiert dazu genutzt werden, ihre europapolitischen Positionen darzustellen. Werden die Merkmale zusammengeführt, dann ergibt sich das folgende Bild: Anhand der Programmatik lässt sich für die SPD konstatieren, dass für diese Partei die Europawahl 2009 die Funktion einer Nebenwahl erfüllt. Ein schwacher bis mäßiger Nebenwahleffekt trifft für die Grünen, die Liberalen und auch für DIE LINKE zu. Anhand der Programmatik von CDU und CSU lässt sich hingegen nur ein schwacher Nebenwahlcharakter feststellen. 
Tabelle 7: $\quad$ Der Nebenwahlcharakter deutscher Europawahlprogrammatik

\begin{tabular}{|c|c|c|c|c|c|c|}
\hline $\begin{array}{l}\text { Merkmale } \\
\text { Nebenwahl }\end{array}$ & CDU & CSU & SPD & FDP & $\begin{array}{l}\text { DIE } \\
\text { LINKE }\end{array}$ & $\begin{array}{l}\text { Bündnis 90/ } \\
\text { Die Grünen }\end{array}$ \\
\hline $\begin{array}{l}\text { Geringerer } \\
\text { Ressourceneinsatz: } \\
\text { Länge d. Programme }\end{array}$ & $X$ & $X$ & $X$ & $X$ & $X$ & $X$ \\
\hline $\begin{array}{l}\text { Geringerer } \\
\text { Ressourceneinsatz: } \\
\text { Anzahl involvierter } \\
\text { Parteiakteure } \\
\end{array}$ & - & - & $X$ & - & - & - \\
\hline $\begin{array}{l}\text { Dominanz des } \\
\text { nationalen Framings }\end{array}$ & - & - & - & - & - & - \\
\hline $\begin{array}{l}\text { Ähnliches Ranking } \\
\text { der wichtigsten } \\
\text { Politikbereiche }\end{array}$ & - & - & $X$ & $x^{\prime}$ & $d x_{0}^{\prime}$ & 次 \\
\hline $\begin{array}{l}\text { Größere ideologische } \\
\text { Distanz zwischen den } \\
\text { Parteien }\end{array}$ & $X$ & $X$ & $X$ & $X$ & $X$ & $X$ \\
\hline Nebenwahlcharakter & schwach & schwach & stark & mäßig & mäßig & mäßig \\
\hline
\end{tabular}

\section{Einfluss des nationalen Parteienwettbewerbs}

Die eingangs hergeleitete Hypothese beinhaltet die Vermutung, dass der Nebenwahleffekt mit dem Status als Regierungs- oder Oppositionspartei korrespondiert. Werden alle Merkmale gleichzeitig berücksichtigt, dann ergibt sich insgesamt ein sehr uneinheitliches Bild. Während der Nebenwahlcharakter für die Wahlprogrammatik der Sozialdemokraten stark ausgeprägt ist - und damit der Vermutung widerspricht -, lässt sich für ihre Koalitionspartner (CDU, CSU) nur ein schwacher Nebenwahleffekt konstatieren. Der Nebenwahlcharakter der Europawahlprogramme ist bei den Oppositionsparteien mäßig ausgeprägt, woraus sich schließlich kein klarer Zusammenhang ableiten lässt. Die Hypothese ist daher eher zu verwerfen.

Bei einer Fokussierung auf die programmatischen Inhalte zeigen sich durchaus Effekte in Abhängigkeit von den Rollen im deutschen Parteienwettbewerb: Die Anteile des europäischen Framings sind bei der SPD und CDU am 
höchsten. Die CSU nimmt eine gewisse Sonderrolle ein, da sie sich als Regionalpartei profilieren will. Der hohe Anteil des EU-Framings kann dahingehend interpretiert werden, dass insbesondere europäische Themen in den Mittelpunkt gerückt werden sollen, um mögliche Unzulänglichkeiten der Regierungsarbeit nicht auf die Agenda zu setzen. Auf der anderen Seite zeigt eine qualitative Bewertung des Europawahlprogramms der Grünen, dass der relativ hohe Anteil des nationalen Framings auffällig aus der Kritik an der amtierenden deutschen Regierung resultiert. Werden auch bei den anderen Parteien die Anteile des gesamtnationalen Framings berücksichtigt, zeigen sich ein geringer Anteil bei SPD und DIE LINKE und ein sehr hoher Prozentsatz bei der CDU sowie der CSU. Abgesehen von dem bereits angesprochenen Effekt der regionalen Profilierung bei der CSU verdeutlicht das recht unterschiedliche Framing der Regierungsparteien das Dilemma der Koalitionspartner: Während die Sozialdemokraten relativ stark in der Kritik standen, umschiffte die CDU unter ihrer Parteivorsitzenden Angela Merkel relativ geschickt die Kritik an ihrer Regierungsarbeit, sodass ein höherer EU-Bezug als taktisches Ausweichmanöver kaum nötig war. Dieser etwas genauere Blick auf das Framing verdeutlicht, dass der Status als Regierungs- bzw. Oppositionspartei durchaus die Ausprägung einzelner Merkmale beeinflussen kann, sich jedoch nicht auf den Nebenwahlcharakter der Europawahlprogrammatik als solcher auswirken muss.

\section{Fazit}

Für die Nachfrageseite wurde hinlänglich gezeigt, dass die Wahlen zum Europäischen Parlament für die deutschen Wähler Nebenwahlen darstellen. Für die deutschen Parteien lag hingegen bisher keine systematische Konzeption eines möglichen Nebenwahlcharakters der Europawahlen vor. Dieses Kapitel schließt diese Lücke anhand einer Analyse der Europa- und Bundestagswahlprogramme im Jahr 2009.

Die empirischen Ergebnisse zeichnen ein differenziertes Bild. Hinweise auf einen Nebenwahlcharakter der Europawahlen konnten für alle Parteien gefunden werden, allerdings variiert das Ausmaß erheblich. Zunächst zeigt sich für alle Parteien, dass sie zum einen weniger Ressourcen für ihre Europa- als für ihre Bundestagswahlprogramme einsetzen. Zum anderen ist die Polarisierung der Parteien auf der Links-Rechts-Achse in den Europawahlprogrammen stärker ausgeprägt.

Unterschiedlich sind jedoch jene Merkmale des Nebenwahlcharakters ausgeprägt, die sich auf die Inhalte der Wahlprogramme beziehen. So variiert der 
Nebenwahleffekt auf die Themensetzung als auch auf den Anteil des europäischen Framings der deutschen Wahlprogramme. Hervorzuheben ist jedoch, dass die deutschen Parteien ihre Europawahlprogrammatik sehr wohl in den europäischen Kontext setzen und damit das Merkmal einer Dominanz des nationalen Framings nicht erfüllt ist. Darin liegt ein wesentlicher Unterschied zwischen der Nachfrage- und Angebotsseite bei den Europawahlen. Demnach reagieren die Parteien - zumindest in ihren wahlprogrammatischen Inhalten - hier nicht auf das Wählerverhalten. Vielmehr vermitteln sie ihre europapolitischen Ansichten und Ideale, um so möglicherweise auch ihre Kompetenz für diese mittlerweile so relevante Entscheidungsebene zu signalisieren. 


\section{Literaturverzeichnis}

Binder, Tanja; Wüst, Andreas M. (2004): Inhalte der Europawahlprogramme deutscher Parteien 1979-1999. In: Aus Politik und Zeitgeschichte, B 17, 38-45.

Braun, Daniela; Salzwedel, Maike; Stumpf, Christian; Wüst, Andreas M. (2007): Euromanifesto Documentation. Mannheim: MZES.

Budge, Ian (2001): Validating the Manifesto Research Group Approach. Theoretical Assumptions and Empirical Confirmations. In: Laver, Michael (Hrsg.) (2001): Estimating the Policy Positions of Political Actors. London, New York: Routledge, $50-65$.

Budge, Ian; Klingemann, Hans-Dieter; Volkens, Andrea; Bara, Judith; Tanenbaum, Eric (Hrsg.) (2001): Mapping Policy Preferences. Estimates for Parties, Electors, and Governments 1945-1998. Oxford, New York: Oxford University Press.

Budge, Ian; Robertson, D.; Hearl, D. (Hrsg.) (1987): Ideology, Strategy and Party Movement: Spatial Analysis of Post-War Election Programmes in 19 Democracies. Cambridge: Cambridge University Press.

Ferrara, Frederico; Weishaupt, J. Timo (2004): Get Your Act Together - Party Performance in European Parliament Elections. In: European Union Politics, 5 (3), 283-306.

Gabel, Matthew J.; Hix, Simon (2002): Defining the EU Political Space - An empirical Study of the European Elections Manifestos, 1979-1999. In: Comparative Political Studies, 35 (8), 934-964.

Heath, Anthony; McLean, Iain; Taylor, Bridget; Curtice, John (1999): Between first and second order. A comparison of voting behaviour in European and local elections in Britain. In: European Journal of Political Research, 35 (3), 389-414.

Hix, Simon (2001): Legislative Behaviour and Party Competition in the European Parliament. An Application of Nominate to the EU. In: Journal of Common Market Studies, 39 (4), 663-688.

Hix, Simon; Marsh, Michael (2007): Punishment or protest? Understanding European Parliament Elections. In: Journal of Politics, 69 (2), 495-510.

Hix, Simon; Noury, Abdul; Roland, Gerard (2005): Power to the Parties: Cohesion and Competition in the European Parliament, 1979-2001. In: British Journal of Political Science, 35 (2), 209-234.

Kreppel, Amie; Hix, Simon (2003): From "grand coalition" to left-right confrontation Explaining the shifting structure of party competition in the European Parliament. In: Comparative Political Studies, 36 (1-2), 75-96.

Kritzinger, Sylvia; Cavatorta, Francesco; Chari, Raj S. (2004): Continuity and Change in Party Positions towards Europe in Italian Parties. An Examination of Parties' Manifestos. In: Journal of European Public Policy, 11 (6), 954-974.

Laver, Michael; Budge, Ian (Hrsg.) (1992): Party Policy and Government Coalitions. Houndsmills, Basingstoke, Hampshire: The MacMillan Press.

Marsh, Michael (1998): Testing the Second-Order Election Model after Four European Elections. In: British Journal of Political Science, 28 (4), 591-607. 
Marsh, Michael (2005): The Results of the 2004 European Parliament Elections and the Second-Order Model. In: Niedermayer, Oskar and Schmitt, Hermann (Hrsg.) (2005): Europawahl 2004. Wiesbaden: VS Verlag für Sozialwissenschaften, 142158.

Poguntke, Thomas (2007): Europeanization in a Consensual Environment? German Political Parties and the European Union. In: Poguntke, Thomas, Aylott, Nicholas, Carter, Elisabeth, Ladrech, Robert and Luther, Kurt Richard (Hrsg.) (2007): The Europeanization of National Political Parties: Power and organizational adaption. London, New York: Routledge, 108-133.

Reif, Karlheinz; Schmitt, Hermann (1980): Nine Second-Order National Elections - A Conceptual Framework for the Analysis of European Election Results. In: European Journal of Political Research, 8 (1), 3-44.

Schmitt, H. (2005): The European parliament elections of June 2004: Still second-order? In: West European Politics, 28 (3), 650-679.

Schmitt, Hermann (1996): Germany: A Bored Electorate. In: van der Eijk, Cees and Franklin, Mark N. (Hrsg.) (1996): Choosing Europe? The European Electorate and National Politics in the Face of Union. Ann Arbor: The University of Michigan Press, 137-156.

Schneider, Daniel; Rössler, Patrick (2005): Von Straßburg über Brüssel nach Erfurt - und zurück? Die Second-Order-Wahl zum Europaparlament in der MehrfachWahlsituation in Thüringen. In: Tenscher, Jens (Hrsg.) (2005): Wahl-Kampf um Europa. Analysen aus Anlass der Wahlen zum Europäischen Parlament 2004. Wiesbaden: VS Verlag für Sozialwissenschaften, 212-234.

Sigalas, Manos; Mokre, Monika (2010): Coordination between National and European Parties. Preliminary Evidence from the 2009 European Elections. ECPR Joint Sessions of Workshops. Münster.

Tenscher, Jens (2005): Mit halber Kraft voraus! Parteikampagnen im Europawahlkampf 2004. In: Tenscher, Jens (Hrsg.) (2005): Wahl-Kampf um Europa. Analysen aus Anlass der Wahlen zum Europäischen Parlament 2004. Wiesbaden: VS Verlag für Sozialwissenschaft, 30-55.

Trechsel, Alexander H.; Mair, Peter (2009): When Parties (also) Position Themselves: An Introduction to the EU Profiler. Working Paper RSCAS 2009/65. Florenz: EUI

van der Brug, Wouter; van der Eijk, Cees; Schmitt, Hermann; Marsh, Michael; Franklin, Mark N.; Thomassen, Jacques; Semetko, Holli A.; Bartolini, Stefano (2007): European Elections, Domestic Politics and European Integration. In: van der Brug, Wouter and van der Eijk, Cees (Hrsg.) (2007): European Elections \& Domestic Politics. Lessons from the Past and Scenarios for the Future. Notre Dame: University of Notre Dame Press, 226-261.

Van der Eijk, Cees; Franklin, Mark N. (Hrsg.) (1996): Choosing Europe? The European Electorate and National Politics in the Face of Union. Ann Arbor MI: University of Michigan Press.

Volkens, Andrea (2002): Manifesto Coding Instructions (Second Revised Edition). Discussion Paper FS III 02-201. Berlin: Wissenschaftszentrum Berlin für Sozialforschung (WZB). 
Weber, Till (2007): Campaign Effects and Second-Order Cycles: A Top-Down Approach to European Parliament Elections. In: European Union Politics, 8 (4), 509-536.

Wüst, Andreas M. (2009): Parties in European Parliament Election: Issues, Framing, the EU, and the Question of Supply and Demand. In: German Politics, 18 (3), 426-440.

Wüst, Andreas M.; Roth, Dieter (2005): Parteien, Programme und Wahlverhalten. In: Tenscher, Jens (Hrsg.) (2005): Wahl-Kampf um Europa. Analysen aus Anlass der Wahlen zum Europäischen Parlament 2004. Wiesbaden: VS Verlag für Sozialwissenschaften, 56-85.

Wüst, Andreas M.; Schmitt, Hermann (2007): Comparing the Views of Parties and Voters in the 1999 Election to the European Parliament. In: van der Brug, Wouter and van der Eijk, Cees (Hrsg.) (2007): European Elections and Domestic Politics. Notre Dame: University of Notre Dame Press, 73-93.

Wüst, Andreas M.; Volkens, Andrea (2003): Euromanifesto Coding Instructions. Working Paper. Mannheim, Mannheimer Zentrum für Europäische Sozialforschung. 